Canadian Oncology

Nursing Journal

Revue canadienne

de soins infirmiers

en oncologie

Volume 29, Issue 1 - Winter 2019

elSSN: 2368-8076 


\title{
L'infirmière praticienne, un rôle parfaitement adapté à la pratique des soins palliatifs : étude descriptive qualitative
}

\author{
par Carmel M. Collins et Sandra P. Small
}

L 'approche des soins palliatifs consiste à s'occuper avec bienveillance des personnes souffrant de maladies menaçant leur vie, afin de maintenir la meilleure qualité de vie qui soit (Hawley, 2017). Cela implique : une gestion efficace des symptômes pour régler les manifestations physiologiques des maladies; des interventions pour promouvoir le mieux-être social, spirituel et émotionnel des patients et de leurs familles; des soins en fin de vie de haut niveau pour maintenir les patients dans le confort et la dignité à l'approche de la mort (Organisation mondiale de la Santé, 2011; Worldwide Palliative Care Alliance [WPCA], 2014). On recommande les soins palliatifs non seulement pour le cancer, mais aussi pour les maladies chroniques menaçant la vie, comme l'insuffisance cardiaque ou rénale ou diverses maladies neurologiques (Hawley, 2017). Vu la prévalenfigurece élevée et la hausse prévue des maladies chroniques, non seulement au Canada, mais aussi dans le monde entier, le besoin de soins palliatifs est déjà grand et le sera encore plus dans l'avenir (Association canadienne des soins palliatifs [ACSP], 2014; Agence de la santé publique du Canada, 2016; WPCA, 2014).

Même s'il est souhaitable d'intégrer les soins palliatifs tôt dans la gestion des maladies chroniques menaçant la vie, cela n'arrive pas si souvent (Hawley, 2014). Beaucoup de gens qui pourraient en bénéficier n'en reçoivent pas du tout, ou seulement en toute fin de vie (Hawley, 2017). Au Canada, seulement $30 \%$ des mourants reçoivent des soins palliatifs (ACSP,

\section{AU SUJET DES AUTEURES}
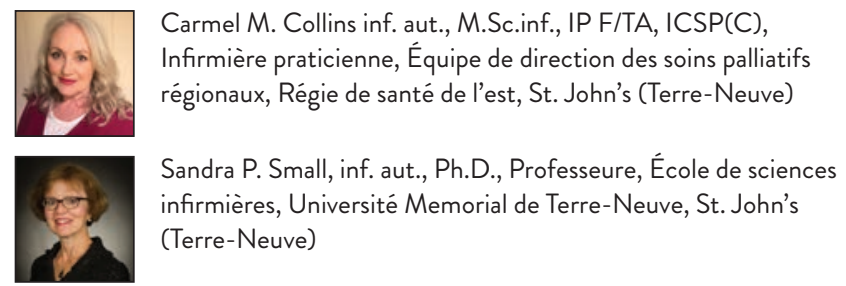

Carmel M. Collins inf. aut., M.Sc.inf., IP F/TA, ICSP(C), Infirmière praticienne, Équipe de direction des soins palliatifs régionaux, Régie de santé de l'est, St. John's (Terre-Neuve)

Sandra P. Small, inf. aut., Ph.D., Professeure, École de sciences infirmières, Université Memorial de Terre-Neuve, St. John's (Terre-Neuve)

Auteure-ressource : Carmel M. Collins, 100 Forest Road, bureau 328, Southcott Hall, St. John's (Terre-Neuve) A1A 1E5 Tél. : 709-777-8039

Courriel : carmelduggancollins@gmail.com

Conflit d'intérêts

Les auteures déclarent l'absence de tout conflit d'intérêts.

Financement

Cette étude a reçu l'appui financier de l'École de sciences infirmières de l'Université Memorial de Terre-Neuve ainsi que de l'Association des infirmières de Terre-Neuve-et-Labrador (Association of Registered Nurses of Newfoundland and Labrador).

DOI: $10.5737 / 236880762911016$

2014). Plusieurs facteurs contribuent au problème d'accessibilité : accès limité à des spécialistes (professionnels de la santé comme des médecins ou des infirmières autorisées ayant reçu une formation spéciale et vécu une expérience clinique de terrain), formation inadéquate des praticiens généralistes (médecins de famille, des infirmières spécialisées en santé communautaire, etc.), manque de ressources financières pour les services de soins palliatifs, distance pour les patients vivant en milieu rural ou éloigné (ACSP, 2014; Société canadienne des médecins de soins palliatifs, 2016; Hawley, 2017).

Selon certaines études, les infirmiers et infirmières praticiennes (IP) pourraient combler en partie cet écart dans les services de soins palliatifs (Deitrick et al., 2011). Elles sont en effet des infirmières autorisées dotées d'une formation complémentaire et d'expérience clinique (Association des infirmières et infirmiers du Canada [AIIC], 2016; Initiative canadienne sur les infirmières et infirmiers praticiens, 2006). En tant qu'infirmières en pratique avancée (IPA), elles ont l'autonomie et la compétence nécessaires pour diagnostiquer des troubles, demander et interpréter des tests diagnostiques, prescrire des médicaments, procéder à des interventions cliniques avancées et gérer des maladies tout en travaillant dans un cadre de collaboration interprofessionnelle et de soins infirmiers holistiques centrés sur les patients. Les IP exercent dans des milieux de soins variés comme des hôpitaux, des cliniques communautaires et des établissements de soins de longue durée, ainsi qu'en milieu urbain, rural et éloigné. Par conséquent, au-delà des IP spécialisés en soins palliatifs ou travaillant spécifiquement dans des centres de soins palliatifs, les IP en général semblent aussi tout indiqués pour fournir des soins aux personnes souffrant de maladies chroniques menaçant leur vie, y compris à un stade avancé. Voilà pourquoi cette étude cherche à répondre à la question suivante : «Quel rôle jouent, dans l'offre de soins palliatifs, les infirmiers et infirmières praticiennes qui ne sont pas spécialisés en soi dans ce domaine et qui ne travaillent pas non plus dans des établissements spécialisés dans la prestation de ces soins? »

\section{REVUE DE LA LITTÉRATURE}

On en sait peu sur la pratique de l'infirmier ou infirmière praticienne en soins palliatifs à l'extérieur des centres spécialisés dans ces soins. La recherche indique toutefois que dans ces centres, les IP contribuent aux soins palliatifs en jouant plusieurs rôles : ils gèrent les troubles médicaux, consultent d'autres prestataires de soins sur la condition des patients, fournissent aux patients et aux familles un soutien psychologique (counseling) et de l'éducation (sur l'état 
de santé, le traitement, etc.), coordonnent les services aux patients, fournissent des soins continus et représentent les patients (Deitrick et al., 2011; Mitchell et al., 2016; O'Connor, Palfreyman, Le, et Lau, 2016; Osborn et Townsend, 1997; Williams et Sidani, 2001).

Bien que peu d'études aient été trouvées sur les conséquences qu'avait sur les patients la pratique des IP en contexte de soins palliatifs, il semble que le rôle de ce professionnel des soins de santé ait des répercussions positives. Des prestataires de soins de santé d'établissements de soins de longue durée ont souligné que les IP avaient amélioré et accéléré l'accès aux interventions palliatives, ce qui avait permis de transférer moins de résidents dans des hôpitaux pour obtenir ces soins (Kaasalainen et al., 2013). De même, des infirmières, des médecins et d'autres intervenants ont indiqué que les services de soins palliatifs offerts par des IP s'étaient traduits par une amélioration des soins auprès des patients en phase terminale, moins d'hospitalisation, plus de décès à la maison, et un meilleur soutien à domicile pour les proches aidants (Mitchell et al., 2016). Une IP travaillant dans un centre d'oncologie offrant des soins palliatifs a signalé que les patients étaient mieux informés et composaient mieux avec la maladie, avaient une meilleure qualité de vie, suivaient mieux le plan de soins prescrit et étaient plus satisfaits des soins reçus, contrôlaient bien leurs symptômes et s'assuraient une bonne prévention des complications (Williams et Sidani, 2001).

Les résultats qualitatifs de ces études correspondent aux conclusions des évaluations de la pratique IP en soins palliatifs. Une réflexion prospective de l'une des fonctions en milieu hospitalier et communautaire indiquait que la pratique de l'IP contribuait à réduire les visites aux urgences et les admissions dans les hôpitaux, de même que la durée des séjours à l'hôpital pour les patients en soins palliatifs (O'Connor et al., 2016). La rétroaction de patients et de leurs proches aidants indiquait que les soins de l'IP augmentaient leur confiance pour la gestion à domicile. Dans une autre étude reposant sur l'examen des dossiers médicaux, la pratique d'une IP dans une clinique de première ligne offrant des soins palliatifs a permis de réduire le nombre de visites des patients aux urgences et d'améliorer les scores symptomatiques chez certains patients (Owens et al., 2011).

Même si les IP peuvent faire une différence auprès des patients en soins palliatifs, il est important de noter qu'elles ne sentent pas toutes à l'aise (Tyree, Long, et Greenberg, 2005) ou en confiance (Dahlin, Coyne, et Cassel, 2016; Letizia et Jones, 2012) pour offrir ces interventions. Une vaste étude réalisée auprès d'IP de divers domaines de pratique pour examiner leur rôle dans les soins en fin de vie a révélé que la majorité pensait qu'une formation et expérience de travail plus poussées sur les soins en fin de vie les aiderait à mieux communiquer avec les patients et leurs familles sur les questions de soins en fin de vie (Tyree et al., 2005). Dans une autre étude, les infirmières en pratique avancée (incluant les IP) avaient indiqué un manque de confiance pour offrir des soins palliatifs avant de participer à un programme de formation continue intensif sur le sujet, confiance qui s'était grandement accrue après le programme (Dahlin et al., 2016). De même, des IP travaillant dans des établissements de soins longues durées disaient, dans une proportion de $61 \%$, manquer de confiance pour évaluer ou gérer des patients en soins palliatifs avant de suivre un programme spécialisé. Après cette formation continue, $83 \%$ se sentaient plus à l'aise (Letizia et Jones, 2012). Soulignons plus particulièrement que les infirmières en pratique avancée (incluant les IP) signalaient avoir reçu peu de contenu sur les soins palliatifs pendant leurs études universitaires (Dahlin et al., 2016; Letizia et Jones, 2012). De même, les professeurs d'un grand nombre de programmes, tout en reconnaissant l'importance de former sur les soins en fin de vie, jugeaient que le contenu à ce sujet dans leurs programmes était au mieux moyennement adéquat et efficace (Paice et al., 2006a, 2006b).

\section{MÉTHODES ET PROCÉDURES}

Cette étude a été menée au moyen des méthodes descriptives qualitatives proposées par Sandelowski (2000). Une description qualitative exige une collecte de données narratives pour décrire entièrement le sujet de recherche en fonction de la perspective des participants à l'étude. L'étude a été approuvée par un comité d'éthique de la recherche et le consentement éclairé a été obtenu pour tous les participants.

\section{Participants}

L'étude s'est déroulée dans une province de l'est du Canada. Les participants étaient des IP autorisés n'exerçant pas prioritairement en soins palliatifs, mais pouvant avoir des patients en soins palliatifs (les IP spécialisés en soins palliatifs ou travaillant dans un établissement spécialisé dans ces soins n'étaient pas admissibles). Le recrutement s'est fait par courriel, tous les IP admissibles de la région géographique ciblée ayant été sollicités. Onze participants se sont présentés ainsi, et un sondage en boule de neige a permis d'en ajouter huit autres. Ces 19 IP se répartissaient ainsi : 16 en région urbaine et 3 en région rurale; 1 homme et 18 femmes; 17 en pratique familiale/tous âges (soins de première ligne pour toutes les populations et étapes de la vie, prévention et soins) et 2 auprès d'une clientèle adulte (centré sur les adultes malades); 5 avec un diplôme d'infirmière autorisée seulement avant de devenir IP, 7 avec plutôt un seul baccalauréat en sciences infirmières avant, et 7 avec une maîtrise en sciences infirmières axée IP. Les participants avaient entre 32 et 62 ans (moyenne 48,5 ans), cumulaient entre 7 et 41 ans de pratique infirmière (moyenne 26,1 ans) et exerçaient comme IP depuis 4 mois à 18 ans (moyenne 9,4 ans).

Du point de vue des soins palliatifs, les IP formaient deux groupes distincts : I3 rencontraient des patients en soins palliatifs dans leur pratique et leur offraient des soins, et 6 n'avaient aucun patient en soins palliatifs. Les IP du premier groupe exerçaient dans des cliniques en santé communautaire de première ligne ou aux urgences en y offrant des soins à toutes sortes de patients, y compris à des patients souffrant de maladies chroniques ou menaçant leur vie, ou encore dans des établissements de soins de longue durée offrant des soins en gériatrie. Ils avaient l'occasion d'offrir des soins palliatifs 
occasionnellement ou souvent. Les autres IP qui n'avaient pas de patients en soins palliatifs exerçaient soit dans des unités hospitalières de soins actifs ou dans des cliniques spécialisées où les populations de patients n'incluaient pas des patients en soins palliatifs.

\section{Collecte de données et analyse}

Les données ont été recueillies par la première auteure, elle-même IP spécialisée en soins palliatifs. Avant d'entamer la collecte de données, elle a réfléchi à ce qu'elle savait, pensait et ressentait au sujet du rôle de l'infirmière praticienne en soins palliatifs, et a consigné ses réflexions dans un journal. Elle a régulièrement passé en revue ses réflexions à mesure qu'elle recueillait et analysait les données dans le but d'éviter les préjugés ou toute interprétation forcée des données. Une entrevue semi-structurée complète a été menée auprès de chaque participant à l'aide de questions ouvertes, afin d'obtenir de l'information sur les expériences vécues par les IP, leurs perspectives et leur point de vue sur la pratique IP en soins palliatifs. Voici quelques exemples des questions posées : «Qu'est-ce que l'expression "soins palliatifs" évoque pour vous? », " Comment voyez-vous le rôle de l'IP dans la pratique des soins palliatifs? », etc. Des pistes de réflexion et commentaires étaient formulés pour obtenir des détails. Les entrevues, enregistrées sur support numérique, se déroulaient en privé et ont été retranscrites mot à mot. Dix-sept entrevues ont été menées en personne et deux au téléphone. Après chaque entrevue, l'auteure a noté dans son journal de recherche les observations faites pendant l'entrevue ainsi que ses réflexions sur ce que l'IP avait partagé. Ces réflexions resservaient dans les entrevues subséquentes et ont aidé les chercheuses à mieux analyser les données ensuite.

L'analyse a été menée de concert par les deux chercheuses au moyen des procédures d'analyse du contenu qualitatif des interventions (Graneheim et Lundman, 2003; Sandelowski, 2000), en même temps que la collecte de données. Cette approche permettait de faire ressortir immédiatement l'essentiel de chaque entrevue et alimentait les entrevues subséquentes, qui pouvaient être orientées pour compléter au mieux les données. Les données de chaque entrevue ont été codées et catégorisées. Une synthèse des catégories établies dans l'ensemble des entrevues a fait ressortir les grands thèmes; la saturation thématique a été atteinte après 19 participants. Le processus analytique a été facilité par la comparaison continuelle des codes et catégories à l'intérieur d'une entrevue et entre celles-ci, par le cumul de citations des participants (en utilisant des pseudonymes pour les distinguer), et la schématisation des thèmes (voir figure 1).

\section{RÉSULTATS}

Les constats tournaient tous autour d'un thème central, le fait que le rôle de l'IP est « parfaitement adapté » à la pratique des soins palliatifs. L'une des participantes, Bertha, l'a exprimé ainsi : « [La pratique des soins palliatifs] est un domaine parfait pour l'infirmière praticienne [...] semblant fait sur mesure pour ce rôle. " Pour certains IP, cette opinion se fondait sur leur expérience professionnelle d'offre de soins à des patients en soins palliatifs dans leur pratique; pour d'autres, qui n'avaient pas cette expérience, cette opinion se fondait sur leur connaissance de la « nature même du travail d'infirmière praticienne » (Bertha), du simple fait d'être soi-même IP. Certains facteurs facilitants et d'autres contraignants ont été relevés.

Le rôle de facilitateur est l'un des attributs de l'IP et se décline en trois thèmes :

1. Pratique autonome et à large portée - Les participants savaient que les patients en soins palliatifs avaient besoin de soins médicaux complexes et étaient donc particulièrement à l'écoute pour veiller aux « mesures de confort [...] et de contrôle de la douleur » (Emma). Ils ont fait ressortir plusieurs caractéristiques spécifiques aux IP concernant l'autonomie et la large portée de leur pratique, qui conviennent vraiment bien pour que les patients en soins palliatifs aient une « meilleure qualité de vie » (Kate). Ces caractéristiques dépassent grandement ce que les infirmières autorisées peuvent faire et impliquent des compétences et des connaissances poussées pour évaluer, diagnostiquer et traiter des troubles médicaux et être en mesure d'intervenir dans la gestion des symptômes, de prescrire des médicaments (notamment des médicaments contrôlés comme les opioïdes), de gérer tous les aspects des soins et de fournir des soins continus. Rita a ainsi fait allusion au fait que les IP pouvaient répondre aux besoins complets des patients en soins palliatifs : « Ce serait tellement bien si une infirmière praticienne pouvait aller visiter la personne [en soins palliatifs] et s'occuper de tous ses besoins, quels qu'ils soient; ce serait l'idéal ».

2. Pratique adaptée - Les IP ont conclu que leur pratique était bien adaptée aux soins palliatifs, car les patients nécessitant ces soins se trouvent en contexte hospitalier et ambulatoire, là où exercent les IP, en marge des établissements spécialisés en soins palliatifs et en oncologie. Les établissements de soins de longue durée en gériatrie et les cliniques en milieu rural ont notamment été ciblés comme secteurs de pratique

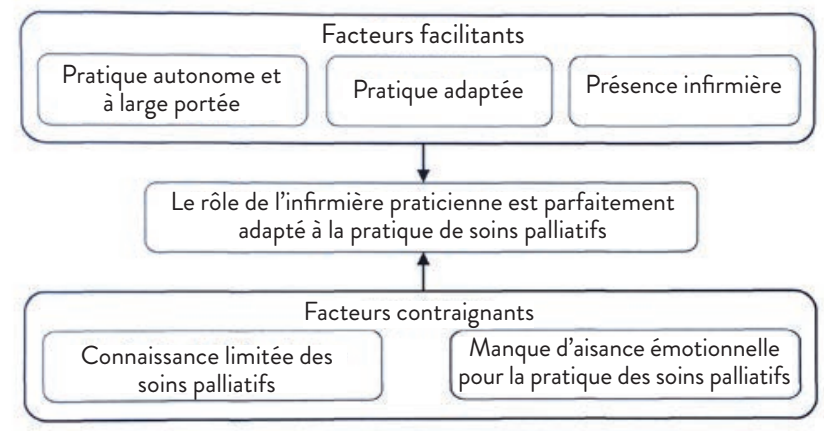

Figure 1 : Représentation thématique des facteurs facilitants et contraignants pour un rôle d'IP parfaitement adapté à la pratique des soins palliatifs. Les facteurs facilitants sont des attributs associés au rôle d'IP (contexte de pratique autonome et à large portée, pratique adaptée et présence infirmière), tandis que les facteurs contraignants ont trait à des attributs personnels des IP (connaissance limitée des soins palliatifs et manque d'aisance émotionnelle pour la pratique de ces soins). 
où les IP sont particulièrement susceptibles de rencontrer des patients nécessitant des soins palliatifs puisque les premiers accueillent invariablement des patients en soins palliatifs en raison de la prévalence des maladies chroniques mettant en danger la vie des personnes âgées qui s'y trouvent, et on ne trouve généralement pas de services spécialisés en soins palliatifs dans les deuxièmes, ce qui oblige les patients à s'en remettre à leur prestataire de soins de première ligne pour ces soins. Dans les deux contextes, l'IP peut s'avérer le principal (voire le seul) prestataire de soins de santé. Ces conditions font des IP des professionnels de choix pour les soins palliatifs. Voici comment Sarah a décrit la situation unique de l'IP pour offrir des soins de première ligne en milieu rural :

Surtout à la campagne, où il n'y a pas d'équipe [...] On est léquipe [...] le prestataire de soins [qui] met les plans en place, [...] qui suit [les patients en soins palliatifs] à toutes les étapes, [...] dose les médicaments, [...] éduque et offre un soutien psychosocial.

3. Présence infirmière - Les IP étaient d'avis que la « présence infirmière » auprès des patients et de leurs familles était essentielle en soins palliatifs, et que les IP ont peuvent, avec leurs compétences, apporter cette présence qui s'incarne notamment dans leur approche holistique, et fait d'eux des intervenants parfaits pour les soins palliatifs. Être présent, c'est savoir se monter disponible, à l'écoute et attentif. C'est « être là, simplement et totalement » (Lynn). Le rôle propre aux IP d'offrir directement des soins continus assure en effet leur disponibilité et leur présence. Leur écoute et leur attention se reflètent dans les besoins physiques et psychosociaux auxquels ils répondent pour optimiser le mieux-être des patients. Cette force de présence des IP s'ancre dans leurs compétences en « communication avancée », leurs techniques « d'écoute active » et leur aptitude pour « l'empathie ». Les IP ont souligné l'importance de la présence pour développer une relation de «confiance » entre le prestataire des soins de santé et le patient en soins palliatifs et sa famille, ce qui est particulièrement important pour avoir des conversations ouvertes et honnêtes sur la maladie, le pronostic et les soins en fin de vie, et ultimement pour aider les patients et leurs familles à trouver « une zone de confort » psychologique (Mary). Comme l'a exprimé Sally, être présent rend possible le dialogue avec les patients sur « leur spiritualité, leur foi, leur soutien familial. [...] Ont-ils accompli ce qu'ils devaient accomplir dans cette vie-ci? Ont-ils l'impression qu'il leur manque quelque chose [...] ou en ont-ils juste assez d'être malades et aimeraient en finir? »

Les obstacles qui empêchent l'IP d'être apte à offrir des soins palliatifs sont ses attributs personnels, qui se répartissent en deux thèmes :

1. Connaissance limitée des soins palliatifs - Les IP avaient la perception qu'une connaissance « spécialisée » des soins palliatifs était nécessaire pour offrir des soins palliatifs et que sans ces connaissances spécialisées, leur savoir en la matière demeurait limité et les rendait moins aptes dans ce domaine. Ils estimaient en outre que cette spécialisation s'acquérait dans le cadre d'études universitaires formelles et par formation continue, ainsi qu'en travaillant dans le domaine. Il est intéressant de noter qu'ils ont souligné que leurs études universitaires d'IP n'abordaient que très peu la question des soins palliatifs ou encore n'accordaient pas suffisamment d'importance au rôle de l'IP dans « la gestion [...] du patient en soins palliatifs » (Emma). À leur avis, «ce qui a été enseigné dans notre programme [d'IP] n'était pas assez [pour maîtriser les soins palliatifs]. Devenir IP n'outille absolument pas sur comment prendre réellement soin de quelqu'un en soins palliatifs. On se sent donc plutôt nerveux [à l'idée d'offrir ces soins] » (Kate).

Par conséquent, il n'est pas surprenant que les IP qui, dans cette étude, n'avaient pas de patients en soins palliatifs aient indiqué avoir peu de connaissances sur le sujet. Parmi les autres, certains affichaient une bonne base de connaissances même si leurs études universitaires officielles d'IP, disaientils, « ne les avaient certainement pas préparés à prendre soin de patients en soins palliatifs » (Tina). Ces IP attribuaient leurs connaissances sur le sujet à l'expérience acquise au fil du temps auprès de patients en soins palliatifs et en consultant d'autres professionnels avec qui ils collaborent ou des spécialistes du domaine. Certains avaient par ailleurs profité de la formation continue (ex. congrès ou conférences) pour accroître leurs connaissances. Les IP ont souligné la valeur et la confiance acquises en pouvant consulter des personnes renseignées pour obtenir du soutien dans leur prise de décision sur la façon de gérer au mieux les patients en soins palliatifs. « Je pouvais toujours appeler et discuter de la gestion de la douleur avec quelqu'un de l'équipe des soins palliatifs [...] J'ai toujours senti que je pouvais appeler lorsque la gestion d'un symptôme me laissait perplexe. C'était toujours une bonne chose. » (Mary) Certains IP ont laissé entendre qu'avoir accès spécifiquement à un autre IP spécialisé en soins palliatifs serait particulièrement utile pour obtenir des conseils et guider leur pratique dans ce domaine.

Les autres IP avaient une expérience limitée auprès de patients en soins palliatifs, n'en ayant rencontré que quelquesuns dans le cadre de leur pratique, et trouvaient donc qu'ils avaient une connaissance limitée des soins palliatifs. Ils reconnaissaient avoir besoin de "plus d'information et de connaissances » (Marie) dans ce champ d'exercice, particulièrement sur « les protocoles et directives cliniques, et la pratique fondée sur l'expérience clinique » (Sally). Ils ont ainsi fait ressortir qu'ils pourraient tirer profit d'enseignements complémentaires pour prescrire des opioïdes, offrir des soins en fin de vie, et discuter des décisions à prendre sur les soins en fin de vie avec les patients et les familles. Bien que ces IP reconnaissaient la valeur de la formation continue pour améliorer la pratique, ils n'en avaient pas encore suivi en soins palliatifs. De même, tout en reconnaissant l'importance de consulter des experts en soins palliatifs pour renforcer leur assurance et leur confiance dans cette pratique, certains remarquaient ne pas avoir accès à ce type de soutien ou n'en pas connaître l'existence. 
Qu'ils aient participé ou non à une formation continue en soins palliatifs, la plupart des IP prodiguant des soins dans ce domaine trouvaient que le système de soins de santé offrait peu de soutien, en général, pour couvrir les dépenses et l'absence au travail pour suivre une formation. Par exemple, les IP souhaitant suivre des activités de formation doivent parfois utiliser leurs vacances pour ce faire. En raison du soutien limité qu'offre le système, certains concluaient que la seule façon pour eux de se former sur le sujet était par la lecture autonome d'articles de revues scientifiques ou sur Internet.

2. Inconfort émotionnel en pratique de soins palliatifs - Les IP estimaient que travailler en soins palliatifs demandait un certain tempérament, à savoir une aisance émotionnelle pour aider des patients et familles qui font face à des troubles pouvant mener à la mort. L'aptitude à agir comme IP en soins palliatifs varie donc en fonction de l'aisance de l'IP à offrir ces soins. Les soins palliatifs ne seraient donc pas «faits pour tout le monde » et ceux qui « ne sont pas à l'aise » préféreront vraisemblablement travailler avec des populations n'ayant pas besoin de ce type de soins. Certains des IP qui n'offraient pas de soins palliatifs ont d'ailleurs révélé qu'ils seraient mal à l'aise d'en offrir en raison du défi émotionnel que travailler avec des patients vivant ces tristes situations impose.

À l'inverse, les IP qui étaient impliqués dans la pratique de soins palliatifs avaient au moins un certain niveau d'aisance émotionnelle pour offrir ces soins. Ces IP entretenaient une philosophie personnelle sur la mort qui contribuait à leur aisance : la mort est une étape «normale » dans la vie et «mourir fait aussi partie de la vie » (Joan). Leur niveau de confort était néanmoins aussi affecté par l'étendue de leur expérience clinique dans le domaine. D’ailleurs, les IP ayant davantage d'expérience disaient être émotionnellement très à l'aise pour offrir ces soins. Selon eux, travailler avec des patients en soins palliatifs et leurs familles était un privilège et ils en tiraient une grande satisfaction, comme l'a relaté Rita :

Lorsqu'on sait qu'on peut aider quelqu'un à mieux vivre une expérience, ça paraît [...] Ça nous aide à nous sentir bien quand on peut [...] aider une personne et faciliter les choses pour une autre; c'est là qu'on sait qu'on a bien fait notre boulot.

\section{DISCUSSION}

Les IP dans cette étude n'étaient pas des spécialistes des soins palliatifs et ne travaillaient pas dans des établissements spécialisés en soins palliatifs, mais qu'ils aient ou non fourni des soins palliatifs à des patients, tous pensaient que le rôle de l'IP convenait parfaitement à cette pratique. Cet avis est conforme aux résultats d'autres études indiquant que les IP contribuaient grandement aux soins palliatifs (Kaasalainen et al., 2013; Mitchell et al., 2016; O’Connor et al., 2016; Owens et al., 2011). Des auteurs sont parvenus à la même conclusion, c'est-à-dire que les IP, en raison de leur expérience et de leur formation, sont tout à fait aptes à répondre aux besoins primaires et à offrir des soins palliatifs aux patients en fin de vie (Owens et al., 2011), et qu'ils étaient probablement, au sein du personnel hospitalier, les mieux placés pour intervenir auprès des patients en fin de vie (Tyree et al., 2005).

Les IP dans cette étude ont avancé que leur rôle convenait particulièrement bien à la pratique en soins palliatifs pour trois grandes raisons : a) la pratique autonome et à large portée permet à l'IP de traiter et de gérer les troubles médicaux des patients en soins palliatifs pour qu'ils aient une meilleure qualité de vie; b) la pratique adaptée donne l'occasion d'offrir des soins palliatifs; et c) la présence infirmière permet aux IP d'être attentifs à tous les besoins des patients, y compris à leurs besoins psychosociaux, pour assurer un mieux-être optimal. La gestion médicale comme fonction de l'IP en soins palliatifs a aussi été identifiée dans d'autres études (Deitrick et al., 2011; Kaasalainen et al., 2013; Mitchell et al., 2016; O'Connor et al., 2016; Osborn et Townsend, 1997). Bien qu'on en sache peu sur la pratique de l'IP auprès de patients en soins palliatifs dans des établissements autres que des unités de soins palliatifs, les résultats d'une étude sur les patients en soins palliatifs d'établissements de soins longue durée ont indiqué que les IP étaient bien placés pour offrir et promouvoir des soins palliatifs optimaux aux résidents et à leurs familles (Kaasalainen et al., 2013). Une autre étude, dans la continuité du point de vue révélé ici que la présence infirmière est cruciale en soins palliatifs et inhérente à la pratique holistique des IP, confirme qu'en se montrant présents auprès de leurs patients, les IP peuvent fournir le soutien psychosocial si important en soins palliatifs. Les IP passent en effet beaucoup de temps avec leurs patients, les conseillent et créent un environnement favorisant la confiance (Deitrick et al., 2011). Des membres du personnel et des familles des établissements de soins longue durée ont aussi rapporté la présence des IP, qui prennent le temps d'écouter et de soutenir les familles en soins palliatifs (Kaasalainen et al., 2013).

La présence infirmière a été largement examinée dans la littérature comme phénomène fondamental de la pratique infirmière (Easter, 2000; Finfgeld-Connett, 2006; McMahon et Christopher, 2011). On l'identifie dans les théories infirmières comme une qualité humaniste et une façon d'être dans la relation infirmière-patient qui permet à l'infirmière de comprendre les expériences propres à chaque patient, afin de lui offrir des soins personnalisés (Parse, 1992; Paterson et Zderad, 1978; Sitzman et Watson, 2014). En raison de son caractère essentiel en soins infirmiers, il n'est pas surprenant que les IP reconnaissent la présence infirmière comme fondamentale dans leur pratique et la manifestent dans leurs interactions avec les patients et leurs familles.

Bien que les IP dans cette étude estimaient que ce rôle convenait parfaitement à la pratique des soins palliatifs, ils considéraient aussi que cette adéquation était freinée lorsque l'IP manquait de connaissances ou d'aisance émotionnelle dans ce domaine. Indépendamment de leur titre professionnel (inf. aut., B.Sc.inf. ou M.Sc.inf.), les IP soulignaient tous avoir reçu une formation très limitée en soins palliatifs pendant leurs études universitaires. Ce manque dans les études 
de premier et deuxième cycles a aussi été rapporté dans d'autres études (Dahlin et al., 2016; Letizia et Jones, 2012; Paice et al., 2006a, 2006b). Les IP qui, dans cette étude, maîtrisaient bien les soins palliatifs ont énoncé que cela résultait de l'expérience acquise sur le terrain, de la pratique collaborative et consultative avec des professionnels aguerris et, pour certains, de la participation à des activités de formation continue. Il n'est que logique de constater que les IP n'ayant acquis que très peu d'expérience en soins palliatifs, suivi aucune formation continue ou reçu aucun soutien en soins palliatifs avaient par conséquent une connaissance limitée du sujet. Ces derniers étaient par ailleurs bien conscients de la nécessité d'acquérir plus de connaissances en la matière, ce qui concorde avec les résultats d'autres études où les IP reconnaissaient avoir besoin d'en savoir plus sur les soins palliatifs, se sentir peu ou pas à l'aise d'offrir ces soins (Dahlin et al., 2016; Letizia et Jones, 2012) et avoir besoin de plus de formation et d'expérience sur les soins en fin de vie pour mieux communiquer avec les patients et leurs familles à ce sujet (Tyree et al., 2005). Aucune étude n’a été trouvée sur la contribution du soutien consultatif pour les IP en soins palliatifs. Par ailleurs, l'opinion des IP comme quoi le recours à la formation continue améliorerait leur pratique en soins palliatifs concorde avec celle d'IP dans d'autres études, lesquels se sentaient plus à l'aise après avoir suivi une formation continue sur le sujet pour offrir des soins palliatifs (Dahlin et al., 2016) et amorcer une discussion sur la fin de vie (Tyree et al., 2005), ou encore avaient modifié leur pratique après avoir acquis de nouvelles connaissances en soins palliatifs (Letizia et Jones, 2012).

Les IP dans cette étude pensaient que l'expérience clinique a aussi de l'importance pour développer un confort émotionnel lorsqu'on travaille avec des patients et des familles faisant face à la mort. Aucune étude portant sur le confort émotionnel en soins palliatifs n'a été trouvée. Toutefois, comme dans les résultats de la présente étude, où certains IP étaient mal à l'aise d'offrir des soins palliatifs en raison des défis émotionnels que cela impose, d'autres études ont fait ressortir que des infirmières généralistes travaillant dans des hôpitaux et établissements communautaires (sans soins palliatifs) auraient vécu des difficultés émotionnelles en offrant des soins aux patients en phase terminale (Bloomer, Endacott, O'Connor, et Cross, 2013; Wallerstedt et Andershed, 2007; Wiegel, Parker, Fanning, Reyna, et Gasbarra, 2007). Tout comme les IP qui, dans cette étude, étaient confortables avec les soins palliatifs de par leur philosophie sur la mort et éprouvaient une satisfaction à s'occuper de patients en soins palliatifs, les infirmières ayant choisi de travailler dans les établissements de soins palliatifs étaient elles aussi très engagées dans leur travail, y trouvaient un but et se montraient résilientes, ce qui donne à entendre qu'il faut un certain type de personnalité pour offrir des soins palliatifs (Ablett et Jones, 2007). Par conséquent, comme l'ont fait ressortir les IP dans cette étude et d'autres études, il est possible que la pratique en soins palliatifs ne convienne pas à toutes les infirmières et infirmiers.

\section{Limite de l'étude et implications pour l'éducation, les politiques et la recherche}

L'échantillon de convenance composé de 19 IP issus d'une même région à l'intérieur d'une province canadienne constitue une limite de cette étude. La diversité d'IP (divers milieux de travail, en milieu urbain ou rural, parcours éducatifs variés et expérience variable en soins palliatifs) ainsi que les procédures de recherche descriptive et qualitative rigoureuses qui ont été suivies renforcent cependant les constats, qui peuvent donc s'ajouter au savoir scientifique sur les IP et les soins palliatifs.

Comme le contenu des programmes d'enseignement des IP est déjà bien volumineux et diversifié, il est irréaliste de s'attendre à une couverture en profondeur des soins palliatifs. Toutefois, un contenu sur les pratiques exemplaires de base paraît approprié, assorti possiblement d'une offre ciblée détaillée sur les soins palliatifs pour les étudiants IP qui prévoient travailler dans des secteurs avec des populations en soins palliatifs, notamment en soins longue durée, en soins de première ligne et dans des établissements spécialisés. En plus des courants de pratique actuels des IP dans les programmes (famille/tous âges, adultes, enfants), les établissements d'enseignement pourraient inclure un volet en soins palliatifs. On suggère en outre que les IP s'engagent de façon continue dans des activités de perfectionnement pour soutenir la pratique des soins palliatifs, par exemple en participant à des activités structurées (congrès, conférences) ou plus libres (lecture d'écrits spécialisés sur le sujet). Les IP qui offrent régulièrement des soins palliatifs pourraient aussi songer à obtenir une certification spécialisée, comme celle de l'AIIC sur les soins infirmiers palliatifs en établissements de soins de santé. Les établissements de soins de santé devraient réserver des ressources financières aux IP pour qu'ils puissent accéder à de la formation continue et à des experts en soins palliatifs qu'ils pourront consulter pour gérer les soins palliatifs des patients dont ils s'occupent.

Lorsqu'ils sont formés adéquatement en soins palliatifs et jouissent d'un confort émotionnel leur permettant de travailler avec des patients en soins palliatifs et leurs familles, il semble que les IP soient parfaitement adaptés à la pratique des soins palliatifs et pourraient donc aider à répondre aux besoins croissants dans ce domaine. L'efficacité des IP (spécialisés ou non) en soins palliatifs n'a cependant pas encore fait l'objet de bien des recherches, et il serait donc utile d'en effectuer pour déterminer quelles sont les retombées sur les patients et le système de soins de santé lorsque les IP offrent ces soins. 


\section{RÉFÉRENCES}

Ablett, J.R., \& Jones, R.S.P. (2007). Resilience and well-being in palliative care staff: A qualitative study of hospice nurses' experience of work. Psycho-Oncology, 16, 733-740. doi:10.1002/ pon. 1130

Agence de la santé publique du Canada (2016). Quel est l'état de santé des Canadiens? Analyse des tendances relatives à la santé des canadiens du point de vue des modes de vie sains et des maladies chroniques. Ottawa, ON : Ministère de la Santé. [en ligne] https://www.canada. ca/fr/sante-publique/services/publications/vie-saine/quel-est-1etat-sante-des-canadiens.html

Association canadienne des soins palliatifs (2014). Fact sheet: Hospice palliative care in Canada. [en ligne] http://www.chpca.net/ media/330558/Fact_Sheet_HPC_in_Canada\%20Spring\%20 2014\%20Final.pdf

Association des infirmières et infirmiers du Canada (2016). The nurse practitioner: CNA position. [en ligne] https:// www.cna-aiic.ca/ /media/cna/page-content/pdf-en / the-nurse-practitioner-position-statement_2016

Bloomer, M.J., Endacott, R., O'Connor, M., \& Cross, W. (2013). The 'disease' of dying: Challenges in nursing care of the dying in the acute hospital setting. A qualitative observational study. Palliative Medicine, 27(8), 757-764. doi:10.1177/0269216313477176pmj.sagepub.com

Canadian Nurse Practitioner Initiative. (2006). Nurse practitioners: The time is now-a solution to improving access and reducing wait times in Canada. Retrieved from http://docplayer.net/5707777-A-solution-toimproving-access-and-reducing-wait-times-in-canada.html

Canadian Society of Palliative Care Physicians. (2016). How to improve palliative care in Canada: A call to federal, provincial, territorial, regional and local decision-makers. Retrieved from http://www. cspcp.ca/wp-content/uploads/2016/11/Full-Report-How-toImprove-Palliative-Care-in-Canada-FINAL-Nov-2016.pdf

Dahlin, C., Coyne, P.J., \& Cassel, J.B. (2016). The advanced practice registered nurses palliative care externship: A model for primary health care education. Journal of Palliative Medicine, 19(7), 753-759. doi:10.1089/jpm.2015.0491

Deitrick, L.M., Rockwell, E.H., Gratz, N., Davidson, C., Lukas, L., Stevens, D., ... Sikora, B. (2011). Delivering specialized palliative care in the community: A new role for nurse practitioners. Advances in Nursing Science, 34(4), E23-E36. doi:10.1097/ ANS.0b013e $318235834 \mathrm{f}$

Easter, A. (2000). Construct analysis of four modes of being present. Journal of Holistic Nursing, 18, 362-377. doi:10.1177/089801010001800407

Finfgeld-Connett, D. (2006). Meta-synthesis of presence in nursing. Journal of Advanced Nursing, 55(6), 708-714. doi:10.1111/ j1365-2648.2006.03961.x

Graneheim, U.H., \& Lundman, B. (2003). Qualitative content analysis in nursing research: Concepts, procedures and measures to achieve trustworthiness. Nursing Education Today, 24, 105-112. doi:10.1016/j.nedt.2003.10.001

Hawley, P.H. (2014). The bow tie model of 21st century palliative care. Journal of Pain and Symptom Management, 47(1), e2-25. doi:10.1016/j.jpainsymman.2013.10.009

Hawley, P. (2017). Barriers to access to palliative care. Palliative Care: Research and Treatment, 10, 1-6. doi:10.1177/1178224216688887

Kaasalainen, S., Ploeg, J., McAiney, C., Martin, L. S., Donald, F., Martin-Misener, R., ... Sangster-Gormley, E. (2013). Role of the nurse practitioner in providing palliative care in long-term care homes. International Journal of Palliative Nursing, 19, 477-485. doi: 10.12968/ijpn.2013.19.10.477

Letizia, M., \& Jones, T. (2012). An educational intervention for nurse practitioners providing palliative care in nursing homes. Journal of
Hospice and Palliative Nursing, 14, 351-357. Retrieved from http:// www.nursingcenter.com/journalarticle?Article_ID=1359588

McMahon, M.A., \& Christopher, K.A. (2011). Toward a midrange theory of nursing presence. Nursing Forum, 46(2), 71-82. doi:10.1111/j.1744-6198.2011.00215.x

Mitchell, G.K., Senior, H.E., Bibo, M.P., Makoni, B., Young, S.N., Rosenberg, J.P., \& Yates, P. (2016). Evaluation of a pilot of nurse practitioner led, GP supported rural palliative care provision. BMC Palliative Care, 15(93). doi:10.1186/s12904-016-0163-y

O'Connor, M., Palfreyman, S., Le, B., \& Lau, R. (2016). Establishing a nurse practitioner model to enhance continuity between palliative care settings. International Journal of Palliative Nursing, 22(12), 581585. Retrieved from http://www.magonlinelibrary.com/toc/ijpn/22/12

Osborn, C.L., \& Townsend, C.H. (1997). Analysis of telephone communication between hospice nurses and a nurse practitioner group. Home Health Care Management a Practice, 9, 52-58. doi:10.1177/108482239700900513

Owens, D., Eby, K., Burson, S., Green, M., McGoodwin, W., \& Isaac, M. (2011). Primary palliative care clinic pilot project demonstrates benefits of a nurse practitioner-directed clinic providing primary and palliative care. Journal of the American Academy of Nurse Practitioners, 24, 52-58. doi:10.1111/j.1745-7599.2011.00664.x

Paice, J.A., Ferrell, B.R., Virani, R., Grant, M., Molloy, P., \& Rhome, A. (2006a). Graduate nursing education regarding end-of-life care. Nursing Outlook, 54, 46-52. doi:10.1016/j.outlook.2005.04.003

Paice, J.A., Ferrell, B.R., Virani, R., Grant, M., Molloy, P., \& Rhome, A. (2006b). Appraisal of the graduate end-of-life nursing education consortium training program. Journal of Palliative Medicine, 9, 353-360. doi:10.1089/jpm.2006.9.353

Parse, R.R. (1992). Human Becoming: Parse's theory of nursing. Nursing Science Quarterly, 5, 35-42. doi:10.1177/089431849200500109

Paterson, J.G., \& Zderad, L.T. (1978). Humanistic Nursing. New York: National League for Nursing.

Sandelowski, M. (2000). Whatever happened to qualitative description? Research in Nursing and Health, 23, 334-340. doi:10.1002/1098-240X(200008)

Sitzman, K., \& Watson, J. (2014). Caring science, mindful practice: Implementing Watson's human caring theory. New York: Springer.

Tyree, T.L., Long, C.O., \& Greenberg, E.A. (2005). Nurse practitioners and end-of-life care: Beliefs, practices, and perceptions. Journal of Hospice and Palliative Nursing, 7, 45-51. Retrieved from http:// journals.lww.com/jhpn

Wallerstedt. B., \& Andershed, B. (2007). Caring for dying patients outside special palliative care settings: Experiences from a nursing perspective. Scandinavian Journal of Caring Sciences, 21, 32-40. doi: 10.1111/j.1471-6712.2007.00430.x/full

Weigel, C., Parker, G., Fanning, L., Reyna, K., \& Gasbarra, D.B. (2007). Apprehension among hospital nurses providing end-of-life care. Journal of Hospice and Palliative Nursing, 9(2), 86-91. doi:10.1097/01. NJH.0000263530.37671.b8

Williams, D., \& Sidani, S. (2001). An analysis of the nurse practitioner role in palliative care. Canadian Journal of Nursing Leadership, 14(4), 13-19. doi:10.12927/cjnl.2001.19131

Wong, S.L., Gilmour, H., \& Ramage-Morin, P.L. (2016). Alzheimer's disease and other dementias in Canada. Health Reports, 27(5) 11-16. Retrieved from http://www.statcan.gc.ca/pub/82003-x/2016005/article/14613-eng.pdf

World Health Organization. (2011). Palliative care for older people: Better practices. Retrieved from http://apps.who.int/iris/ bitstream/10665/107290/1/e95052.pdf

Worldwide Palliative Care Alliance. (2014). Global atlas on palliative care at the end of life. Retrieved from http://www.thewhpca.org/ resources/global-atlas-on-end-of-life-care 